\title{
Inverse Analysis of Consolidation Behavior with Several Nonlinear Models
}

\author{
Shin-ichi Nishimura ${ }^{1}$ \\ ${ }^{1}$ Graduate School of Environmental and Life Science, 3-1-1 Tsushima-naka, Kita-ku, Okayama 700-8530, Japan \\ E-mail: theg1786@okayama-u.ac.jp
}

\begin{abstract}
The deformation and the pore water pressure prediction of soft ground based on the measured data is the main topic. The inverse analysis technique with the elasto-visco-plastic (EVP) model and proposed stochastic nonlinear model (SNM) are employed. The comparison of the constitutive models, and the variety of measured data are conducted, and an appropriate approach to predict the future consolidation behavior is discussed. In conclusion, SNM could predict future behavior better.
\end{abstract}

Keywords: Consolidation; measurement; prediction; particle filter; inverse analysis; stochastic nonlinear model.

\section{Introduction}

The deformation and the pore water pressure prediction of soft grounds based on the measured data is the main topic. The inverse analysis technique with the elasto-visco-plastic (EVP) model and proposed stochastic nonlinear model (SNM) are employed. The comparison of the constitutive models, and the variety of measured data are conducted, and an appropriate approach to predict the future consolidation behavior is discussed. As the EVP model, the Sekiguchi-Ohta model (1977), which is the very popular in Japan, is employed. To identify the parameters of the EVP model, the particle filter (PF) is employed to overcome severe non-linearity of the stress strain relationship. In Shuku et al. (2012), this approach was successfully applied for the consolidation problems. As an another approach, the SNM developed by the author (Nishimura et al. 2002; 2014; 2016) is also employed. Two approaches are compared here.

To validate the applicability of the proposed methods, model tests were conducted. The interconnected one dimensional consolidation test (Imai 1995) was employed, in which the internal stain and the pore water pressure can be measured simultaneously. Long term settlement behavior from the tests are analyzed, and two types of the methods can be compared precisely. In the PF with EVP model, the four parameters, the compression index, permeability, the coefficient of secondary compression, and the initial volumetric strain rate are selected as unknown variables. While in the SNM, the nonlinear model is selected from the stochastic model based on the random field theory, and the initial Young' modulus, and initial permeability, the coefficient of secondary compression, and initial void ratio rate are identified.

\section{Parameter Identification EVP-Model and PF}

\subsection{EVP-model}

To present the behavior of the soft clay, Sekiguchi-Ohta model is employed as the constitutive equation. The model is a kind of the elasto-visco-plastic model, and evaluate the residual settlement of the clay. The model is described here.

The elasto-visco-plastic volumetric strain is derived by Equation (1), and the yielding function $f$ is defined as Equation (2).

$$
\begin{array}{lll}
\varepsilon_{v}^{v p}=\alpha \ln \left[1+\frac{\dot{V}_{0} t}{\alpha} \exp \left(\frac{f}{\alpha}\right)\right] & \text { (1) } f=\frac{\lambda-\kappa}{1+e_{0}} \ln \frac{p^{\prime}}{p_{0}{ }^{\prime}}+D \eta^{*} & \text { (2) } \\
\Lambda=1-\frac{\kappa}{\lambda} & \text { (4) } \quad \eta^{*}=\sqrt{\frac{3}{2}\left(\frac{s_{i j}}{p^{\prime}}-\frac{s_{i j 0}}{p_{0}^{\prime}}\right)\left(\frac{s_{i j}}{p^{\prime}}-\frac{s_{i j 0}}{p_{0}{ }_{0}}\right)}
\end{array}
$$

In Equation (1)-(5), $\alpha$ is the secondary compression index, $\dot{V}_{0}$ is the initial volumetric strain rate, $t$ is the elapsed time, $\lambda$ is the compression index, $\kappa$ is the swelling index, $D$ is the dilatancy index, $e_{0}$ is the initial void ratio, $p^{\prime}$ is the mean effective stress, $p_{0}{ }^{\prime}$ is the initial mean effective stress, $\mathrm{M}$ is the critical state parameter, $\Lambda$ is irreversibility index . $s_{i j}$ is the deviation stress tensor, and $s_{i j 0}$ is the initial deviation stress tensor.

Proceedings of the 7th International Symposium on Geotechnical Safety and Risk (ISGSR)

Editors: Jianye Ching, Dian-Qing Li and Jie Zhang

Copyright (c) ISGSR 2019 Editors. All rights reserved.

Published by Research Publishing, Singapore.

ISBN: 978-981-11-2725-0; doi:10.3850/978-981-11-2725-0_IS7-4-cd 


\subsection{Algorithm of particle filter}

The particle filter (PF) is the time updating algorithm, in which the probability distribution is approximated by the "particles", namely, discrete samples. This algorithm is applicable for the cases the state variable has strong nonlinearity and non-Gaussian probability distribution, compared with the other filter theories.

In the filter theory, following nonlinear state-space model is introduced.

$$
\begin{aligned}
& \boldsymbol{x}_{t}=F_{t}\left(\boldsymbol{x}_{t-1}\right)+\boldsymbol{v}_{t} \\
& \boldsymbol{y}_{t}=H_{t}\left(\boldsymbol{x}_{t}\right)+\boldsymbol{w}_{t}
\end{aligned}
$$

$$
p\left(\boldsymbol{x}_{t-1} \mid \boldsymbol{y}_{1: t-1}\right)=\frac{1}{N} \sum_{i=1}^{N} \delta\left(x_{t-1}-x_{t-1 \mid t-1}^{(i)}\right)
$$

In Equation (6), the vectors, $\boldsymbol{x}_{t}$ and $\boldsymbol{y}_{t}$ are the state variables and the measured data to present the state at the time series t- $1,2, \ldots, T$, respectively, and present displacements and pore water pressures. The vectors, $\boldsymbol{v}_{t}$ and $\boldsymbol{w}_{t}$ are the system and the observational noises, and $\boldsymbol{v}_{t}=0$ is assumed in this study. The function $F_{t}$ is the nonlinear transient function from time step $t-1$ to $t$, and described by the soil- water coupled finite element analysis system. The function $H_{t}$ is presents the relationship between the state variables and the observed data. If the relationship is linear, the function $H_{t}$ is a matric composed by the elements of 1 and 0 . In Equation (7), $\delta$ is Dirac delta function and $N$ is number of particles.

In PF, the probabilistic distribution of the state variables, is presented by the ensembles of the particles. The particle vectors correspond to the state variables, namely, displacements and pore water pressures. In Equation (7), the filter distribution, $p\left(\boldsymbol{x}_{t-1} \mid \boldsymbol{y}_{1: t-1}\right)$ is described by the ensemble sets at the time step of $t=t-1$, $\left\{\boldsymbol{x}_{t-1 \mid t-1}^{(1)}, \boldsymbol{x}_{t-1 \mid t-1}^{(2)} \cdots, \boldsymbol{x}_{t-1 \mid t-1}^{(N)}\right\}$, and means the conditional probability the occurrence of $\boldsymbol{x}_{t-1}$ under the observed data, $\boldsymbol{y}_{1: t-1}$, which expresses $\left(\boldsymbol{y}_{1}, \boldsymbol{y}_{2}, \ldots, \boldsymbol{y}_{t-1}\right)$. The superscript $(i)$ means the particle number, and subscript, $t-1 \mid t-1$ means the current time step (left) and lastly observation time step (right).

The probability density of the state variable, $\boldsymbol{x}_{t}$ is updated by the following algorithm.

1. The $d$ dimensional state variables vector, $\boldsymbol{x}_{00}^{(i)}$ at the time step, $t=0$, are generated for the particle numbers, $i=1, \ldots, N$. The dimension, $d$ is the number of state variables. The state variables, $\boldsymbol{x}_{00}^{(i)}$ have the initial probability density, $p_{0}(\boldsymbol{x})$, and are the function of $d_{p}$ dimensional vector of the analytical parameters, $\boldsymbol{z}^{(i)}$. The initial weight of the particles is defined as Equation (9).

$$
\boldsymbol{x}_{0 \mid 0}^{(i)}=\boldsymbol{x}_{0 \mid 0}^{(i)}\left(\boldsymbol{z}^{(i)}\right)
$$

$$
\tilde{w}_{0}^{(i)}=1 / N
$$

2. For the time step, $t=1,,,, T$, the following processes (a) (c) are repeated.

a. For each particle, $i$, the time evolution of $x_{t+i}^{(i)}$ is derived by Equations (10) and, the likelihood $l_{t}^{i}$ is evaluated by Equation (11), in which, $p\left(\boldsymbol{y}_{t} \mid \boldsymbol{x}_{t \mid t-1}^{(i)}\right)$ is the probability density function of the observed data, $\boldsymbol{y}_{t}$ under the condition of $\boldsymbol{x}_{t \mid t-1}^{(i)}$.

b. Calculation of Equation (12)

c. Update of the weight of the particle $i$ at time step of $t, \tilde{w}_{t}^{(i)}$ is derived by Equation (13).

$$
\boldsymbol{x}_{t \mid l-1}^{(i)}=F_{t}\left(\boldsymbol{x}_{t-1 \mid k-1}^{(i)}\right) \quad(10) \quad l_{t}^{(i)}=p\left(\boldsymbol{y}_{t} \mid \boldsymbol{x}_{t \mid-1}^{(i)}\right) \quad \text { (11) } \quad W_{t}=\sum_{i=1}^{N} \tilde{w}_{t-1}^{(i)} l_{t}^{(i)} \quad \text { (12) } \quad \tilde{w}_{t}^{(i)}=\tilde{w}_{t-1}^{(i)} l_{t}^{(i)} / W_{t}
$$

If the observation noise, $\boldsymbol{w}_{t}$ obeys the normal distribution, whose covariance function is $\boldsymbol{R}_{t}$, the probability density, $p\left(\boldsymbol{y}_{t} \mid \boldsymbol{x}_{t \mid t-1}^{(i)}\right)$ is expressed by Equation (14), in which $m$ is the dimension of the vector, $\boldsymbol{y}_{t}$.

$$
p\left(\boldsymbol{y}_{t} \mid \boldsymbol{x}_{t \mid t-1}^{(i)}\right)=\frac{1}{(\sqrt{2 \pi})^{m}\left|\boldsymbol{R}_{t}\right|} \exp \left[-\frac{\left(\boldsymbol{y}_{t}-H\left(\boldsymbol{x}_{t \mid-1}^{(i)}\right)\right)^{\mathrm{T}} R_{t}^{-1}\left(\boldsymbol{y}_{t}-H\left(\boldsymbol{x}_{t \mid-1}^{(i)}\right)\right)}{2}\right] \text { (14) } \tilde{\boldsymbol{x}}_{t}=\boldsymbol{x}_{t}\left(\boldsymbol{z}_{t}\right) \quad \text { (15) } \quad \boldsymbol{z}_{t}=\sum_{i=1}^{N} \tilde{w}_{t}^{(i)} z^{(i)}
$$

The prediction of state variable, $\boldsymbol{x}_{t}$ is obtained based on the weighted mean of the parameter vector, $\boldsymbol{z}_{t}$ by Equation (15).

\section{Parameter Identification with Stochastic Nonlinear Model (SNM)}

\subsection{Consolidation model including secondary consolidation}

The settlements of the grounds are calculated by Equation (17), incorporating the secondary consolidation. The settlement at the time $t, S(t)$ is defined the sum of the primary and secondary consolidation settlements, $S_{1}(t)$ and $S_{2}(t)$. The secondary settlement will occur, the critical reduction rate of the void ratio, $\square \dot{e}_{0}$. 


$$
\begin{array}{lll}
S(t)=S_{1}(t) & \left(-\dot{e}_{0}<-\dot{e}\right) \\
S(t)=S_{1}(t)+S_{2}(t) & \left(-\dot{e}_{0} \geq-\dot{e}\right)
\end{array} \quad(17) \quad S_{2}(t)=r_{\alpha} m_{v} H \cdot \ln \left(\frac{t}{t_{\alpha}}\right) \quad(18) \quad t_{\alpha}=\left.t\right|_{\dot{e}=\dot{e}_{0}} \quad \text { (19) } \quad r_{\alpha}=\bar{p} \frac{C_{\alpha}}{C_{c}}
$$

$S_{1}(t)$ is the settlement cased by the dissipation of the pore water pressure, and to evaluate it, the soil - water coupled finite element method is employed. The parameters, which presents the primary consolidation, are the coefficient of volume compressibility, $m_{v}$, and the coefficient of permeability, $k$. The secondary consolidation is defined as Equation (18). In Equation (19), $t_{\alpha}$ is the time corresponding to the time at $\dot{e}_{0}=\dot{e}$, the parameter $r_{\alpha}$ presents the ratio of secondary consolidation defined by Equation (20) for the normally consolidation state. In Equation (20) which, $C_{\alpha}$ is secondary compression index, $C_{c}$ is the compression index, and $\bar{p}$ is the average consolidation stress.

\subsection{Stochastic nonlinear model (SNM)}

Although, the $C_{c}$ method is employed well to present the nonlinearity of the consolidation behaviour, the soft ground often exists in the quasi-overconsolidated state due to the structures of the clay skeleton, and rigidity of the soil changes complicatedly with increase of the consolidation pressure. The nonlinearity of the permeability also necessarily considered. Following procedure is introduced to deal with the nonlinearity of the parameters.

The coefficient of volume compressibility, $m_{v}$, which is the function of the effective major principal stress, $\sigma_{1}^{\prime}$, is expressed as Equations (21) and (22). The effective major principal stress at the end of the primary consolidation, is coincident to the consolidation pressure, $p$ in the consolidation tests. To express the nonlinearity of $m_{v}$, the correction factor, $C_{m v}\left(\sigma_{1}^{\prime}\right)$ is introduced.

$$
m_{v}=C_{m v}\left(\sigma_{1}^{\prime}\right) m_{v I}
$$

$$
C_{m v}\left(\sigma_{1}^{\prime}\right)=m_{v p}\left(\sigma_{1}^{\prime}\right) / m_{v p}\left(\sigma_{1,0}^{\prime}\right)
$$

where $\sigma_{1}{ }^{\prime}$ is the effective major principal stress, $m_{v I}$ is the initial volume compressibility, $\sigma_{1}{ }^{\prime}, 0$ is the initial major effective principal stress, and $m_{\mathrm{v} p}$ is the volume compressibility derived from the prior information.

To consider the reduction of the permeability $k$ with the consolidation process, the following equations are employed.

$k=C_{k}(e) k_{I}$

$$
C_{k}(e)=k_{p}(e) / k_{p}\left(e_{0}\right)
$$

in which $e$ is the void ratio, $k_{I}$ is initial permeability, $e_{0}$ is initial void ratio, and $k_{p}$ is the permeability derived from the prior information. The correction factors, $C_{m v}\left(\sigma_{1}{ }^{\prime}\right), C_{k}(e)$ are ordinary determined from the results standard consolidation tests, $m_{v} \sim \sigma_{1}$ and $k \sim e$ relationships.

In inverse analysis, the Monte Carlo method is applied for the statistical models of the variables, $m_{v p}$ and $k_{p}$, and optimum $C_{m v}$ and $C_{k}$ are determined among the generated samples. The statistical models of $M_{v}=\log m_{v p}$, $K=\log k_{p}$ are defined by the Equations (25) and (26). These models are not necessarily determined from the conducted laboratory tests, and possibly obtained from the database of the consolidation tests for the similar clay.

$$
M_{v}\left(\sigma_{1}{ }^{\prime}\right)=\mu_{M v}\left(\sigma_{1}{ }^{\prime}\right)+\sigma_{M v}\left(\sigma_{1}{ }^{\prime}\right) \xi_{M v}\left(\sigma_{1}{ }^{\prime}\right)
$$

$$
K(e)=\mu_{K}(e)+\sigma_{K}(e) \xi_{K}(e)
$$

in which $\mu_{M v}, \mu_{K}$ are mean functions, $\sigma_{M v}, \sigma_{K}$ are the standard deviation functions, $\xi_{M v}, \xi_{K}$ are $N(0,1)$ type normal random variables including the correlation structures denoted by Equations (27) and (28). The normal random numbers are assigned for the variables, $\xi_{M v}$ and $\xi_{K}$ in the inverse analysis. In the correlation coefficients of Equations (27) and (28), the covariance function, $\operatorname{Cov}(*)$ presents the correlation between two states $(i, j)$ along the effective major principal stress, or the void ratio, and the function, $\exp (*)$ presents the spatial correlation between two points $(i, j)$ along the vertical and horizontal directions.

$r_{M v}\left(\xi_{M v i}, \xi_{M v j}\right)=\operatorname{Cov}\left[\xi_{M v}\left(\sigma_{1, i}^{\prime}\right), \xi_{M v}\left(\sigma_{1, j}^{\prime}\right)\right] \exp \left(-\frac{\left|x_{i}-x_{j}\right|}{l_{x}}-\frac{\left|z_{i}-z_{j}\right|}{l_{z}}\right)$

in which $l_{x}$ and $l_{z}$ are the horizontal and vertical correlation lengths.

$r_{K}\left(\xi_{K i}, \xi_{K j}\right)=\operatorname{Cov}\left[\xi_{K}\left(e_{i}\right), \xi_{K}\left(e_{j}\right)\right] \exp \left(-\frac{\left|x_{i}-x_{j}\right|}{l_{x}}-\frac{\left|z_{i}-z_{j}\right|}{l_{z}}\right)$

in which $\operatorname{Cov}\left[\xi_{M_{v}}\left(p_{i}\right), \xi_{M_{v}}\left(p_{j}\right)\right]$, and $\operatorname{Cov}\left[\xi_{K}\left(e_{i}\right), \xi_{K}\left(e_{j}\right)\right]$ could be determined from the standard consolidation tests or the database. 


\subsection{Parameter identification procedure}

Overall identification algorithm is presented in Figure 1, in which the Monte Carlo method and the nonlinear least square method are mixed. In the first and second steps of the algorithm, the parameters, related to primary and secondary consolidation are identified, respectively. At the first step, the parameters $m_{v I}, k_{I}, C_{m v}$ and $C_{k}$ are identified, and at the second step the parameters $r_{\alpha}$ and $-\dot{e}_{0}$ are identified, and then, the parameters $m_{v I}$ and $k_{I}$ are adjusted again. Although all parameters should be identified simultaneously, two steps approach is conducted for simplicity, since the random fields necessarily determined by Monte Carlo method in the algorithm, this procedure makes identification approach complicated.

In the first step, the squared error $J_{1}$ between calculated and measured values is defined in Equation (29). By minimizing $J_{1}$ with the nonlinear least square method, the parameters, $m_{v I}$ and $k_{I}$ are evaluated. Then, the control parameter, $\lambda$, between the squared errors of the settlement and the pore water pressure, the correlation lengths, $l_{x}$, and $l_{z}$ are changed, and the minimization of $J_{1}$ is repeated. For each trial, the $J_{2}$ value given in Equation (30) is evaluated, for the minimum $J_{2}$, the parameters, $\lambda, l_{x}, l_{z}, C_{m v}$ and $C_{k}$ are finally determined. In the second step, the secondary consolidation parameters are determined by minimizing the $J_{1}$

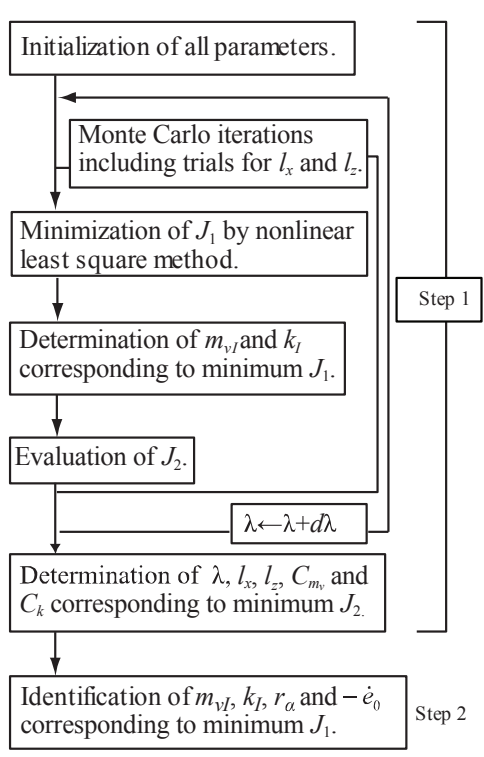

Figure 1. Identification algorithm. again. In Equation (29), $\lambda$ is the control parameter between the displacements and the pore water pressures, $N T$ is the number of time steps, $N N P$ is the number of the displacement observation points, $N E L$ is the number of pore water pressure

Table 1. Soil properties for interconnected consolidation tests observation points, $S_{i}^{j}$ and $\bar{S}_{i}^{j}$ are the calculated and the observed displacements at the observation point $i$ and the time step $j$, and, $u_{i}^{j}$ and $\bar{u}_{i}^{j}$ are the calculated and the observed pore water pressures at the observation point $i$ and the time step $j$, respectively.

$$
\begin{aligned}
& J_{1}=\sum_{j=1}^{N T}\left\{\sum_{i=1}^{N N P}\left(S_{i}^{j}-\bar{S}_{i}^{j}\right)^{2}+\frac{1}{\lambda} \sum_{i=1}^{N E L}\left(u_{i}^{j}-\bar{u}_{i}^{j}\right)^{2}\right\} \\
& J_{2}=\left\{\sum_{j=1}^{N T} \sum_{i=1}^{N N P}\left(S_{i}^{j}-\bar{S}_{i}^{j}\right)^{2}\right\}\left\{\sum_{j=1}^{N T} \sum_{i=1}^{N E L}\left(u_{i}^{j}-\bar{u}_{i}^{j}\right)^{2}\right\}
\end{aligned}
$$

\section{Consolidation Test}

The profile of the test material is presented in Table 1. The test species were formed into $6 \mathrm{~cm}$ in the diameter and $2 \mathrm{~cm}$ in the height from the clay material pre-consolidated at $40 \mathrm{kPa}$. The water content was $86.5 \%$.

Four inter-connected consolidation cells were prepared exhibited in Figure 2. The specimens set in the cells, are denoted as No.1, No.2, No.3 and No.4 from the upper to the bottom. At the upper and the bottom surfaces of each specimen, the settlements and pore water pressures are measured, respectively. The load was applied stepwisely from the $40 \mathrm{kPa}$ to $320 \mathrm{kPa}$. The loading rate, $\Delta p / p$ is 1.0 . The initial void ratios at the step from $80 \mathrm{kPa}$ to $160 \mathrm{kPa}$ is 1.697 .

\section{Analytical Model}

Figure 3 depicts the finite element model studied in this paper. Although in this research, a one-dimensional consolidation is analyzed, for the assumption of the two-dimensional random

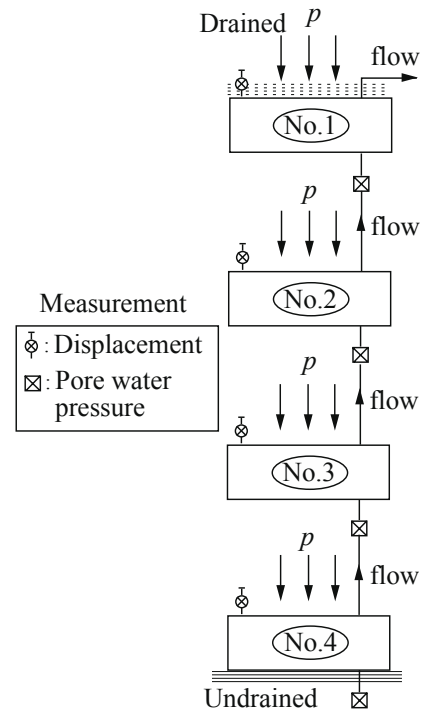

Figure 2. Inter-connected consolidation test 
field of the SNM, two-dimensional model is employed. Since, for the EVP model, the homogeneity is assumed inside each element, the one-dimensional finite element is used. In the one-dimensional case, horizontally single element is employed.

In Figure 3, NP and EL present the points, at which the displacements and pore water pressure are measured. The lateral size of the two-dimensional finite element model is determined to be $4 \mathrm{~cm}$ as the two times value of the maximum lateral correlation length, $2 \mathrm{~cm}$. The consolidation stage of the consolidation pressure from $80 \mathrm{kPa}$ to $160 \mathrm{kPa}$ is analyzed.

Table 2 presents the parameters for the elasto-visco-plastic model. Table 2 also shows the range of the probabilistic parameters for $\alpha, \dot{V}_{0}, \lambda$, and $k$, and the random numbers are generated to obey the uniform distributions for the four parameters.

\section{Analytical Results}

\subsection{Results of EVP with PF}

Figures 4(a) and (b) exhibit the displacements and the pore water pressures, and measured and calculated results are compared. The calculated results are derived from identified parameters by PF based on the all observed data. Although the fitness between observed and calculated behaviours is acceptable, in the early stage of the consolidation, the pore water pressures are overestimated, namely, the calculated pore water pressure is over 80 $\mathrm{kpa}$. The rising up of pore water pressure is supposed to be cause by the in-homogenization of the specimen due to the elasto-plastic behaviour. In the latest stage, the displacements are overestimated. Based on the results, the EVP model might not make the available prediction of the further settlement.

\subsection{Result of SNM}

The detail of the analysis is presented in the previous work (Nishimura et al. 2016). In Figure 5, observed and simulated results by SNM are depicted. As the optimum random field parameters, the values of $l_{x}=0.5 \mathrm{~cm}$, and $l_{z}=0.1 \mathrm{~cm}$ are determined. According to the figure, the displacement is simulated well, and the pore water pressure is also relatively well done. The figure presents the prediction result of the case that the measured data up to $1080 \mathrm{~min}$ are used for the identification, and future behaviour after $1080 \mathrm{~min}$ is well predicted.

\section{Conclusions}

1. In order to consider the strain-dependent behavior of a clay soil, including secondary consolidation, fourcells interconnected consolidation tests were conducted. According to the test results, the settlement shows clear secondary consolidation, and the pore water pressure dissipated faster than with the standard consolidation theory.

2. In the inverse analysis method with the particle filter and the EVP model, the four parameters, the compression index, the permeability, the coefficient of secondary compression, and the initial volumetric strain rate, could be identified from measured displacement and pore water pressure by PF. In the early stage of the consolidation, the pore water pressure was overestimated, and the latest stage, the displacement was overestimated.

3. A stochastic nonlinear model has been introduced to represent the parameter nonlinearity simply, because the complicated constitutive law cannot be accepted for parameter identification. In the model, the nonlinearity of the volume compressibility and the permeability are defined as functions of the effective major principal stress and the void ratio, respectively. The two-dimensional spatial random field was introduced simultaneously.

4. The displacements have been simulated well based on the identified parameters in every case, while the pore water pressure has been simulated well only in the case in which the SNM and the two-dimensional random field are employed simultaneously. Based on the measured settlement and the pore water pressure up to a degree of consolidation of $70 \%$, the future consolidation after $70 \%$ has been predicted

Table 2. Parameters for EVP analysis

\begin{tabular}{|c|c|}
\hline Secondary compression index $\alpha$ & $0.005 \sim 0.015$ \\
\hline $\begin{array}{l}\text { Initial volumetric strain rate } \\
\dot{V}_{0}(1 / \mathrm{min})\end{array}$ & $0 \sim 1.0 \times 10^{-4}$ \\
\hline Compression index $\lambda$ & $0.2 \sim 0.3$ \\
\hline Initial void ratio $e_{0}$ & 1.697 \\
\hline Critical state parameter $\mathrm{M}$ & 1.2 \\
\hline $\begin{array}{c}\text { Consolidation yield stress } \\
\sigma^{\prime}{ }^{\prime}(\mathrm{kPa})\end{array}$ & 80 \\
\hline $\begin{array}{c}\text { Effective overburden stress } \\
\sigma_{v i}^{\prime}(\mathrm{kPa})\end{array}$ & 80 \\
\hline Irreversibility index $\Lambda$ & 0.9 \\
\hline Effective Poisson's ratiov' & 0.3 \\
\hline $\begin{array}{l}\text { Coefficient of permeability } k \\
(\mathrm{~m} / \mathrm{s})\end{array}$ & $\begin{array}{c}1.63 \times 10^{-8} \sim \\
1.63 \times 10^{-6}\end{array}$ \\
\hline $\begin{array}{l}\text { Coefficients of earth pressure at } \\
\text { rest for normally consolidation } \\
\text { and in situ } K_{0}, K_{i}\end{array}$ & 0.5 \\
\hline
\end{tabular}
well. 
5. The inverse analysis with the SNM, could predict the internal settlement and pore water pressure are predicted better than the particle filter with EVP model.

\section{Acknowledgments}

This work was partly supported by JSPS KAKENHI Grant Number $16 \mathrm{H} 02577$.

\section{References}

Imai, G. (1995). Compression and consolidation of clayey soils, 2, 891-935.

Nishimura, S., Shimada, K., and Fujii, H. (2002). Consolidation inverse analysis considering spatial variability and nonlinearity of soil parameters. Soils and Foundations, 42(3), 45-61.

Nishimura, S., Shuku, T., and Fujisawa, K. (2014). Prediction of multidimensional deformation behavior based on observed value, Int., J. of Geomech, 4(3), 04014011 1- 0401401110.

Nishimura, $\overline{\mathrm{S}}$, Shibata, $\overline{\mathrm{T}}$., and Shuku, T. (2016). Prediction of long-term consolidation behavior considering nonlinearity and spatial variability of soil parameters, Proc., of the 6th Asian-Pacific Symposium on Structural Reliability and its Applications, APSSRA2016, 630-635.

Sekiguchi, H. and Ohta, H. (1977). Induced anisotropy and time dependency in clay. Proc., of $9^{\text {th }}$ ICEMFS, 229-239.

Shuku, T., Murakami, A., Nishimura, S., Fujisawa, K., and Nakamura, K. (2012). Parameter identification for Cam Clay model in partial loading model tests using the particle filter. Soils and Foundations, 52(2): 279-298.

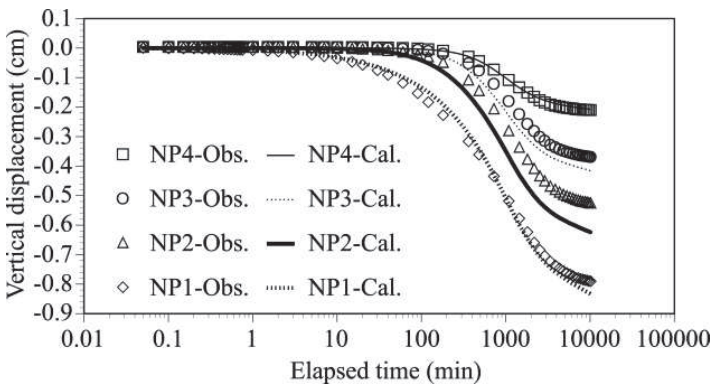

(a) Time-settlement curve

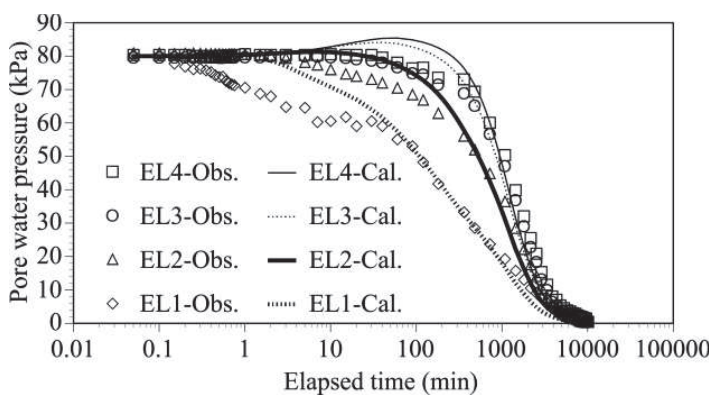

(b) Time-pore water pressure curve

Figure 4. Consolidation behavior observed and calculated by EVP model.

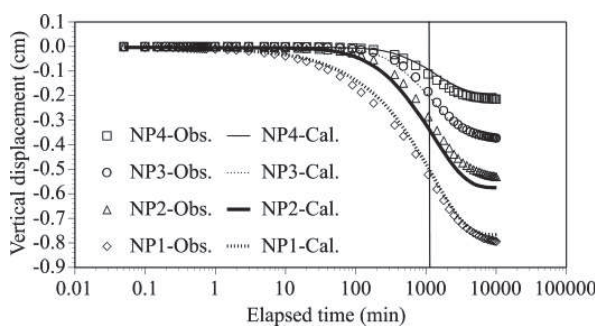

(a) Time-settlement curve.

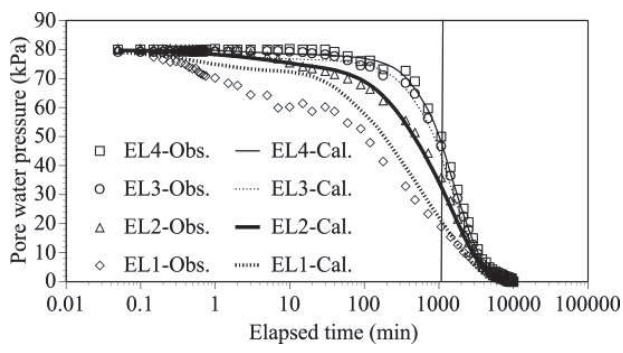

(b) Time-pore water pressure curve.

Figure 5. Consolidation behavior observed and calculated by SNM.

Prediction after $1080 \mathrm{~min}$. 